DRAFT VERSION OCTOBER 29, 2018

Preprint typeset using LTEX style emulateapj v. 04/20/08

\title{
POLARIZATION SIGNATURE OF GAMMA-RAY BURSTS FROM FRAGMENTED FIREBALLS
}

\author{
Davide Lazzati ${ }^{1}$ And Mitchell C. Begelman ${ }^{2,3}$ \\ Draft version October 29, 2018
}

\begin{abstract}
We study the polarization properties of the prompt emission of gamma-ray bursts produced by fragmented fireballs. Such fireballs, known in the literature under various names, are made by the superposition of many individual blobs, each of which produces a spike in the light curve. The differences between pulses are due both to the intrinsic diversity in the blobs' properties and to their orietation with respect to the line of sight. We show that the peak flux and the polarization of each pulse are connected through the orientation of the blob that produces the pulse, while the position angle fluctuates randomly from one pulse to the next and is constant within an individual pulse. The most polarized pulses are those with approximately one tenth the peak flux of the brightest pulse. These conclusions do not depend on the assumed radiation mechanism nor on the energy and Lorentz factor of the blobs. We compare the prediction of this model to a simulated set of observations, showing that a limited sample of GRBs with time-resolved polarization measurements would provide a crucial test for this model. We finally show that a hint of the predicted correlation may have been already observed.
\end{abstract}

Subject headings: gamma-ray: bursts — polarization — radiation mechanisms: non-thermal

\section{INTRODUCTION}

Despite many years of observations, the origin of the prompt emission of gamma-ray bursts (GRBs) is still clouded in mystery. GRB prompt radiation is known to originate within a collimated relativistic outflow with Lorentz factor of several hundreds (Rhoads 1999; Lithwick \& Sari 2001 and references therein). However, the geometry of the outflow, whether it is matter or Poynting flux dominated, the dissipation processes, and the radiation mechanism involved are still matters of open debate.

The amount and the temporal evolution of linear polarization of the prompt GRB emission has been hailed as a key observation to solve, at least, some of these outstanding issues (Eichler \& Levinson 2003; Granot 2003; Lyutikov et al. 2003; Nakar et al. 2003; Waxman 2003; Lazzati et al. 2004a; Lazzati 2006; Dado et al. 2007). On the observational side, the measurement of linear polarization at several hundred $\mathrm{keV}$ has been challenging. An initial report of very high linear polarization in the prompt emission of GRB 021206 ( $\Pi=80 \pm 20 \%$; Coburn \& Boggs 2003) was subsequently shown to be very uncertain, at best (Rutledge \& Fox 2004; Wiggers et al. 2004). Subsequent claims of moderate to high levels of linear polarization in the prompt emission of other GRBs have been plagued by low significance and systematic uncertainties: $\Pi>35 \%$ in GRB 930131 and $\Pi>55 \%$ in GRB 960924 (Willis et al. 2005); $\Pi=98 \pm 33 \%$ in GRB 041219a (Kalemci et al. 2007; McGlynn et al. 2007).

Most of the models proposed to explain the high linear polarization possibly observed predict a constant position angle, since the polarization is expected to lie in the plain that contains both the axis of the jet and the line of sight to the observer. However, a recent observation by Gotz et al. (2009) of GRB 041219a suggests that the polarization angle changes substantially within the burst. Prompted by this observation,

\footnotetext{
Electronic address: davide_lazzati@ncsu.edu

${ }^{1}$ Department of Physics, NC State University, 2401 Stinson Drive, Raleigh, NC 27695-8202

2 Jila, University of Colorado, 440 UCB, Boulder, CO 80309-0440

${ }^{3}$ University of Colorado, Department of Astrophysical and Planetary Sciences, 389 UCB, Boulder, CO 80309-0389
}

we explore in this letter the polarization arising from fragmented fireballs, i.e., fireballs that are made by the superposition of smaller jets or relativistic blobs of material. Within this framework, each pulse of a light curve is produced by a single blob, and the pulse properties depend on the blob intrinsic properties (energy and Lorentz factor) as well as on the orientation between the velocity vector of the blob and the line of sight to the observer. Fragmented fireballs have been proposed in the past under various names as a way to explain GRB prompt emission: shotguns (Heinz \& Begelman 1999), cannonballs (Dado \& Dar 2009 and references therein), subjets (Yamazaki et al. 2006). Additional models that predict a polarization behavior analogous to fragmented fireballs are external shocks on a clumpy medium (Dermer et al. 1999) and precessing jets (Blackman et al. 1996; Portegies Zwart et al. 1999).

This paper is organized as follows: in $\S 2$ we compute the polarization arising from a fragmented fireball, in $\S 3$ we compare the predictions to observations and in $\S 4$ we discuss our results and compare them to the predictions of alternative models.

\section{POLARIZATION FROM A SINGLE FRAGMENT}

Consider a blob with total energy $E$ moving with Lorentz factor $\Gamma$ with an angle $\theta_{o}$ between the velocity vector and the line of sight. Both the radiation flux received at earth and the polarization fraction depend on the viewing angle $\theta_{o}$.

If the blob releases a certain fraction of its energy into radiation, the photon pulse appears brightest for an observer at $\theta_{o}=0$. We call the flux of photons from the on-axis blob in photons per second per square centimeter $\Phi_{\max }$. If the same blob moves with an angle $\theta_{o}>0$, its photon flux scales as

$$
\Phi\left(\theta_{o}\right)=\Phi_{\max } \frac{\delta(\theta)^{3}}{\delta(0)^{3}} \simeq \frac{\Phi_{\max } \delta(\theta)^{3}}{8 \Gamma^{3}}
$$

where $\delta(\theta)=[\Gamma(1-\beta \cos \theta)]^{-1}$ is the Doppler factor.

Consider now radiation produced either by synchrotron emission in a shock-compressed magnetic field (Ghisellini \& Lazzati 1999; Sari 1999; Granot 2003) or by bulk inverse Compton scattering (Begelman \& Sikora 1987; Shaviv \& Dar 
1995, Lazzati et al. 2004). In either case, the radiation has a polarization

$$
\Pi\left(\theta^{\prime}\right)=\Pi_{\max } \frac{1-\cos ^{2} \theta^{\prime}}{1+\cos ^{2} \theta^{\prime}}
$$

where $\theta^{\prime}$ is the angle, in the comoving frame, between the velocity vector and the direction of the photon velocity and $\Pi_{\max }$ is the maximum polarization attainable by the model: $100 \%$ for inverse Compton and $\sim 70 \%$ for synchrotron.

Due to the relativistic aberration, the comoving angles map into observer's angles as

$$
\cos \theta^{\prime}=\frac{\cos \theta-\beta}{1-\beta \cos \theta}
$$

where $\beta$ is the velocity of the fireball in units of the speed of light. Equations 1, 2] and 3 are an implicit system of equations that allow us to plot the fraction $\Pi / \Pi \max$ as a function of $\Phi / \Phi_{\max }$, independently of the fragment energy, radiation mechanism, and Lorentz factor. The result is shown with a thick solid line in Fig. 1. The brightest configuration has no polarization since the fragment points directly to the observer and everything is symmetric. Polarization increases with $\theta_{o}$ until the maximum is reached when $\theta_{o} \sim 1 / \Gamma$ (which corresponds to $\theta^{\prime}=\pi / 2$ in the comoving frame) and $\Phi \sim \Phi_{\max } / 8$.

The analytic result shown above is rigorously valid for a point-like fragment, for which the opening angle $\theta_{j} \ll 1 / \Gamma$. In realistic cases, we expect $\theta_{j} \sim 1 / \Gamma$ or larger, since a hot fragment would expand into the cone of causal connection. We compute the curves of $\Pi / \Pi_{\max }$ versus $\Phi / \Phi_{\max }$ for realistic fragments following Lazzati et al. (2004) and we show the results in Fig. 1. The behavior is analogous, the main difference being a region of low polarization for $\Phi_{\max } / 2<\Phi<\Phi_{\max }$. This is the range of angles for which the line of sight is still inside the fragment, but is not aligned with the fragment axis.

The electric field vector, which defines the position angle of the polarization, lies in the plane that contains both the line of sight to the observer and the velocity vector of the blob. As a consequence, the position angle is constant for an individual pulse. However, since different pulses are due to different blobs that do not share the same velocity vector, the polarization angle should fluctuate randomply from one pulse to the next. Such behavior is not possible in polarization models for non-fragmented fireballs, since the velocity vector in that case maintains the same direction during the whole prompt emission phase.

\section{OBSERVATIONAL CONSIDERATIONS}

The results discussed above allow us to predict the polarization of the pulse from a fragment as a function of the reduction of the peak photon flux with respect to the fragment pointing at the observer. If a GRB fireball were made of identical fragments (identical energy, opening angle, and Lorentz factor) and if at least one of the fragments would always be pointing directly to the observer, polarization measurements from a GRB would lie along one of the lines plotted in Fig. 1

In reality, fragmented fireball models predict fragments with different energies and Lorentz factors and different opening angles. In addition, the brightest observed pulse may not come from a perfectly aligned fragment and therefore the value of $\Phi_{\max }$ may be misidentified. In order to understand the impact of this diversity, we performed a Monte Carlo simulation in which we considered a sample of 30 bursts with the following characteristics. All bursts had 29 fragments with energy randomly distributed between 0.1 and 1 in arbitrary units. The fragments had Lorentz factor randomly distributed between 100 and 400 and opening angle $\theta_{j}=1 / 250=1 /\langle\Gamma\rangle$. All the fragments were ejected within a collimated outflow with opening angle 0.02 radians. In such a configuration, an observer along the axis of the outflow sees on average 4 pulses within $10 \%$ of the peak photon flux.

The result of the simulation are shown in Fig 2] The yellow dots show $\Pi / \Pi_{\max }$ of each individual pulse versus its $\Phi / \Phi_{\max }$. Note that $\Phi_{\max }$ and $\Pi_{\max }$ are calculated for the GRB to which the pulse belongs and not from the theoretical maxima. The yellow dots show a big dispersion around the theoretical curve for a fragment with $\theta_{j}=1 / \Gamma$ (shown with a red solid line) and have very little predictive power. However, the average behavior of the sample is strongly indicative of the underlying mechanism that produces the polarization. Blue symbols show the average value of $\Pi / \Pi_{\max }$ in 10 bins of $\Phi / \Phi_{\max }$. Even though the modulation of the polarization is not as deep as for the point-like blob, a trend of increasing polarization for decreasing peak flux is clearly observable and highly statistically significant (error bars show the standard error of the sample in each bin). Finally, the green line shows the average results that would be obtained with a much larger GRB sample (30000) for which the uncertainty is negligible. Even a large sample does not reproduce the ideal case, mainly due to the low probability of having a blob pointing directly to the observer with only 4 bright pulses in the lightcurve.

The simulation of Fig. 2 shows that even a moderate number of GRBs with reliable time-resolved polarization measurements would represent a crucial test for this model. We should emphasize here that the simulation is made conservatively, since in the real case selection effects would tend to privilege bursts with at least one very bright peak. In such a case, a better evaluation of $\Phi_{\max }$ would be possible. In addition, any burst with more than 4 pulses, would allow for a better determination of $\Phi_{\max }$.

In Fig. 3 we show the polarization results of Götz et al. (2009) overlaid on the model predictions. Even though the error bars are big and strong conclusions cannot be drawn, the observations suggest that the bright peaks are less polarized than the dim ones. However, a systematic effect may produce this result, since dim peaks have less statistics and would produce more uncertain polarization measurements. More robust measurements are necessary to confirm the suggestive Fig. 3 .

\section{DISCUSSION}

We have shown that a fragmented fireball can easily produce highly polarized GRBs with a position angle that fluctuates randomly among individual pulses. In this model, the brightest pulses should be the least polarized, with the maximum polarization observed for pulses with peak photon flux of about $10 \%$ of the peak photon flux of the GRB. These characteristics, possibly observed by Götz et al. (2009) in GRB 041219a, are not reproduced by alternative scenarios:

- Magnetic Domains. Gruzinov \& Waxman (1998) proposed a model in which the magnetic field behind the shock re-organizes into magnetic domains. Within each domain, the field is uniform and gives rise to maximally polarized radiation. Even though the model can explain a randomly fluctuating angle, the maximum polarization expected is of $\Pi \leq 10 \%$, since approximately 100 domains are simultaneously visible to the observer, even if the re-organization proceeds at the speed of light. In addition, this model is contradicted by after- 
glow observations that show a position angle that varies within a small interval (Lazzati et al. 2004b; Greiner 2004).

- 1/Г effects A number of models predict high polarization in the prompt GRB emission as a consequence of a particular viewing geometry (Waxman 2003; Granot 2003; Lazzati et al. 2004a). In such models, the radiation mechanism (either synchrotron or bulk inverse Compton) produces highly polarized radiation in the comoving frame in the direction perpendicular to the direction of motion. In the observer frame, due to relativistic aberration, the highly polarized radiation is observed in a direction that lies at an angle $\theta=1 / \Gamma$ with respect to the velocity vector. If the opening angle of the fireball is small $\left(\theta_{j} \leq 1 / \Gamma\right)$, such an observer could detect polarization up to $100 \%$. However, the polarization position angle lies in the plane that contains the line of sight and the velocity vector, and therefore it can not change during the burst.

- Toroidal magnetic field. If the magnetic field component is dominant in the energy budget of the jet, the GRB radiation is produced by synchrotron emission in a toroidal magnetic field and the polarization can be up to $50 \%$, regardless of the particular configuration of the fireball with respect to the line of sight (Lyutikov et al. 2003). Again, even though this model predicts high linear polarization in most GRBs, it cannot account for position angle variations within a single burst, since the polarization is always directed towards the jet axis.

For these reasons, should high linear polarization with a varying position angle be confirmed by future observations, it would be a strong indication that the fireball producing GRBs is fragmented or, at least, that the energy release is not uniform within the jet opening angle. This models comes with the prediction that the brightest pulses are least polarized, since they are directed straight to the observer. Pulses with approximately $10 \%$ of the peak luminosity should be maximally polarized, since they are directed at an angle $1 / \Gamma$ with respect to the line of sight. As shown in Fig. 2] the prediction will be easily tested when a reasonably large number of GRBs will have observations of linear polarization of their prompt emission.

We thank Gabriele Ghisellini for useful discussions. This work was in part supported by NASA ATP grant NNG06GI06G (DL \& MB) and by Swift GI program NNX08BA92G (DL).

\section{REFERENCES}

Begelman, M. C., \& Sikora, M. 1987, ApJ, 322, 650

Blackman, E. G., Yi, I., \& Field, G. B. 1996, ApJ, 473, L79

Coburn, W., \& Boggs, S. E. 2003, Nature, 423, 415

Dado, S., \& Dar, A. 2009, arXiv:0901.4260

Dado, S., Dar, A., \& De Rujula, A. 2007, arXiv:astro-ph/0701294

Dermer, C. D., Böttcher, M., \& Chiang, J. 1999, ApJ, 515, L49

Eichler, D., \& Levinson, A. 2003, ApJ, 596, L147

Ghisellini, G., \& Lazzati, D. 1999, MNRAS, 309, L7

Götz, D., Laurent, P., Lebrun, F., Daigne, F., \& Bošnjak, Ž. 2009, ApJ, 695, L208

Granot, J. 2003, ApJ, 596, L17

Gruzinov, A., \& Waxman, E. 1999, ApJ, 511, 852

Heinz, S., \& Begelman, M. C. 1999, ApJ, 527, L35

Kalemci, E., Boggs, S. E., Kouveliotou, C., Finger, M., \& Baring, M. G. 2007, ApJS, 169, 75

Lazzati, D. 2006, New Journal of Physics, 8, 131

Lazzati, D., Rossi, E., Ghisellini, G., \& Rees, M. J. 2004a, MNRAS, 347, L1

Lazzati, D., et al. 2004b, A\&A, 422, 121
Lithwick, Y., \& Sari, R. 2001, ApJ, 555, 540

Lyutikov, M., Pariev, V. I., \& Blandford, R. D. 2003, ApJ, 597, 998

McGlynn, S., et al. 2007, A\&A, 466, 895

Nakar, E., Piran, T., \& Waxman, E. 2003, Journal of Cosmology and Astro-Particle Physics, 10, 5

Portegies Zwart, S. F., Lee, C.-H., \& Lee, H. K. 1999, ApJ, 520, 666

Rhoads, J. E. 1999, ApJ, 525, 737

Rutledge, R. E., \& Fox, D. B. 2004, MNRAS, 350, 1288

Sari, R. 1999, ApJ, 524, L43

Shaviv, N. J., \& Dar, A. 1995, MNRAS, 277, 287

Waxman, E. 2003, Nature, 423, 388

Wigger, C., Hajdas, W., Arzner, K., Güdel, M., \& Zehnder, A. 2004, ApJ, 613, 1088

Willis, D. R., et al. 2005, A\&A, 439, 245

Yamazaki, R., Ioka, K., Nakamura, T., \& Toma, K. 2006, Advances in Space Research, 38, 1299 


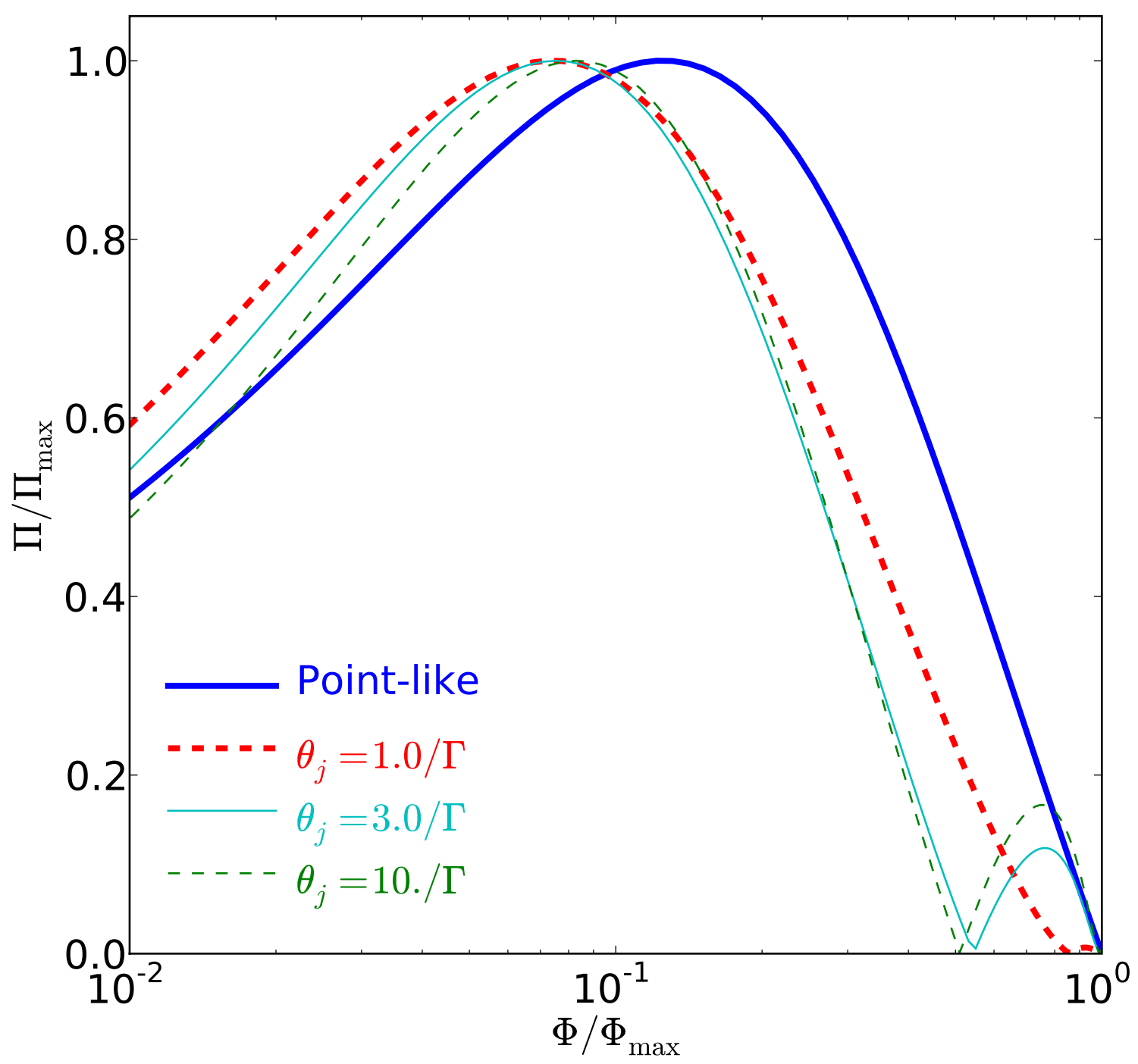

FIG. 1.- Polarization versus photon peak flux for a fragmented fireball. The thick solid line (blue) shows the point-like approximation, for which $\theta_{j}<<1 / \Gamma$. The thick dashed line (red) shows a causally connected fireball $\left(\theta_{j}=1 / \Gamma\right)$, the thin solid line (cyan) shows a fireball with $\theta_{j}=3 / \Gamma$, and the thin dashed line (green) shows a fireball with $\theta_{j}=10 / \Gamma$ ). 


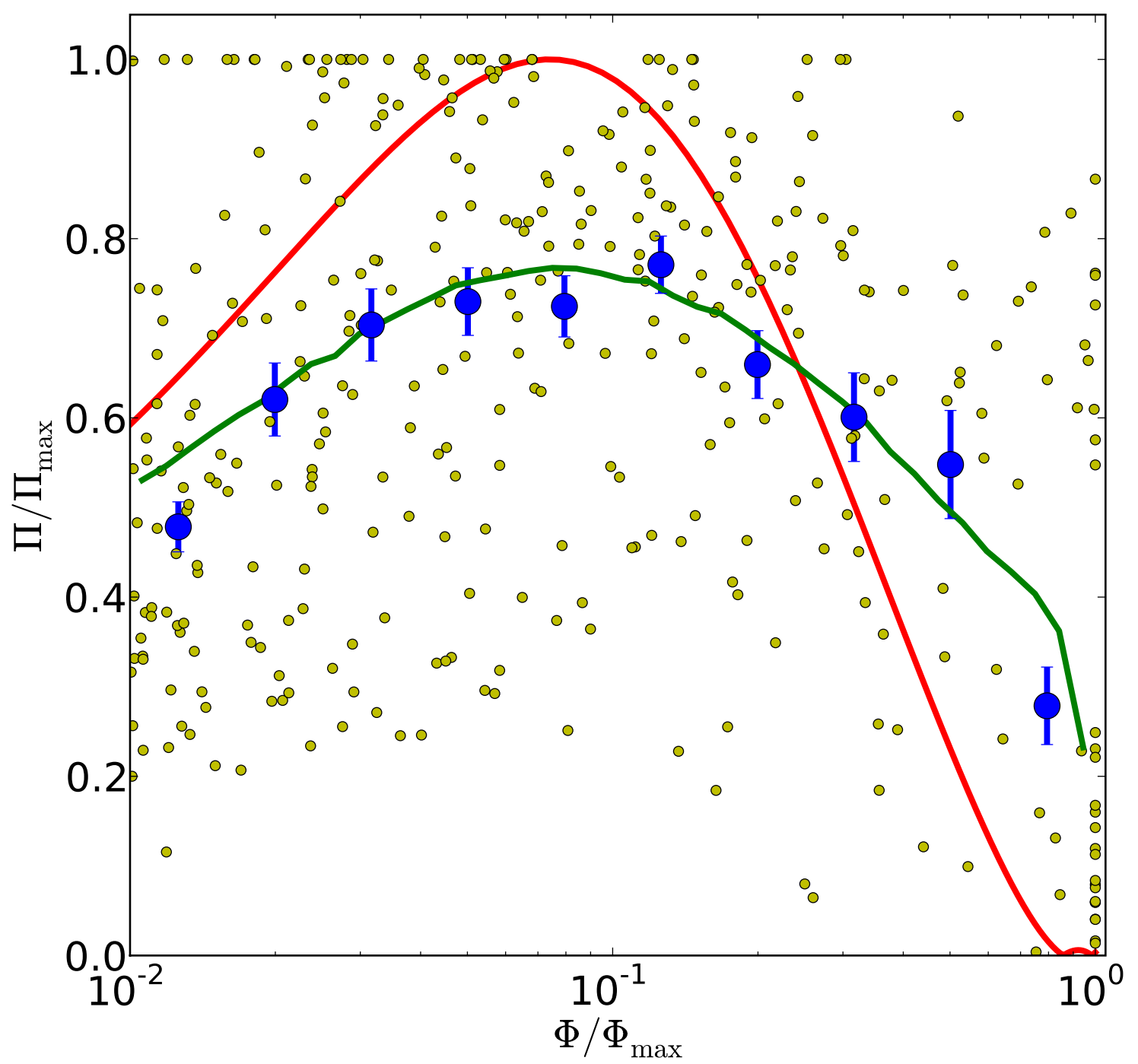

FIG. 2.- Simulation of the observation of a sample of 30 GRBs (yellow circles). Each of them has 5 peaks with peak flux within $10 \%$ of the maximum. The energy of each fragment is randomly distributed between 0.1 and 1 in arbitrary units, the Lorentz factor is randomly distributed between 100 and 400 and each fragment has an opening angle $\theta_{j}=1 / 250=1 /\langle\Gamma\rangle$. The red solid line shows the polarization versus intensity for the point-like approximation. The blue symbols with error bars show the average polarization versus intensity in 10 bins of intensity, while the green solid line shows the result for a very large sample of GRBs. 


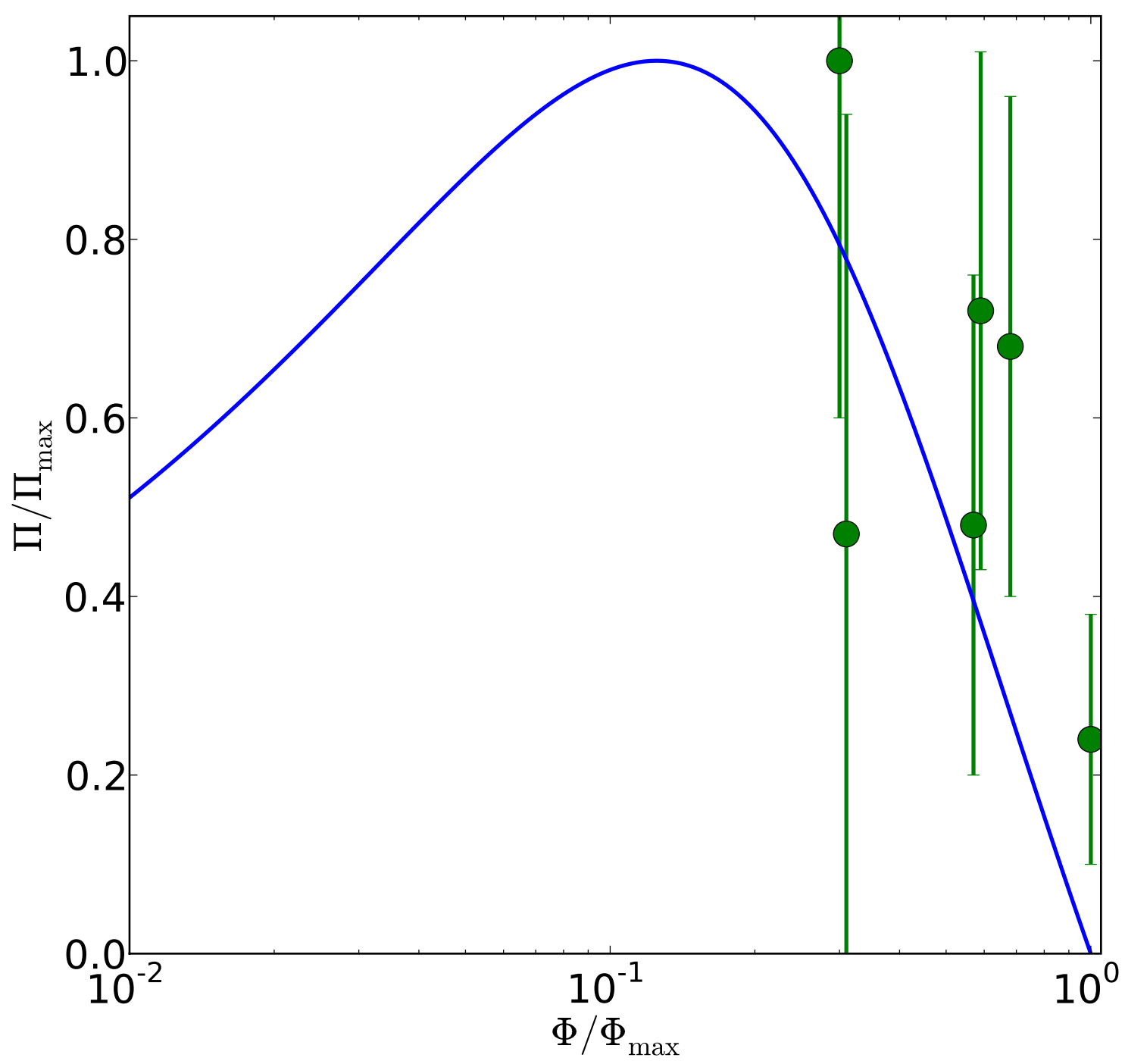

FIG. 3. - Polarized fraction of GRB 041219a from Götz et al. (2009) overlaid on the point-like prediction for the fragmented fireball model. 